\title{
Prenatal effects of maternal nutritional stress and mental health on the fetal movement profile
}

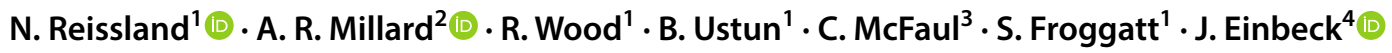

Received: 31 July 2019 / Accepted: 25 April 2020 / Published online: 14 May 2020

(c) The Author(s) 2020

\begin{abstract}
Purpose Prenatal sub-optimal nutrition and exposure to maternal stress, anxiety and depression in pregnancy have been linked to increased postnatal morbidity and mortality. Fetal growth is most vulnerable to maternal dietary deficiencies, such as those evident in hyperemesis gravidarum (HG), early in pregnancy. The purpose of this pilot study was to examine the effects of HG on fetal movement profiles as a measure of fetal healthy development in the 3rd trimester of pregnancy, and to assess whether nutritional stress on the mother can be evaluated using isotopic analysis of hair.

Method We analyzed fetal movement profiles using 4D ultrasound scans at 32- and 36-weeks' gestation. Fetuses of women $(N=6)$ diagnosed with HG, having lost more than $10 \%$ of their body weight in the first trimester of pregnancy were compared to a healthy group $(N=6)$, controlling for stress, depression and anxiety. We tested carbon and nitrogen isotope ratios in maternal hair as a measure of both diet and nutritional changes due to catabolism of body proteins and fats.

Results HG and catabolism were significantly correlated $(p=0.02)$. Furthermore, at 32-weeks' gestation movement profiles of fetuses of mothers with HG differed significantly from the movement profiles of fetuses of healthy mothers. Fetuses of mothers suffering from HG showed a significantly increased ratio of fine-grained movements at 32 weeks $(p=0.008)$; however, there were no significant differences detectable at 36-weeks' gestation.

Conclusion The effect of HG on fetal development as expressed by variations in fetal movement profiles in this pilot study suggest that prenatal effects of HG can be measured using movement profiles. Isotope analysis of hair can supplement this with information on nutritional imbalances early in pregnancy.
\end{abstract}

Keywords Fetal movement profile $\cdot$ 4D ultrasound scans $\cdot$ Hyperemesis gravidarum $\cdot$ Catabolism $\cdot$ Maternal stress

Electronic supplementary material The online version of this article (https://doi.org/10.1007/s00404-020-05571-w) contains supplementary material, which is available to authorized users.

N. Reissland

n.n.reissland@durham.ac.uk

1 Department of Psychology, Durham University, South Road, Durham DH13LE, UK

2 Department of Archaeology, Durham University, South Road, Durham DH1 3LE, UK

3 Child Study Center, Yale Medical School, New Haven, CT, USA

4 Department of Mathematical Sciences, Durham University, South Road, Durham DH1 3LE, UK

\section{Introduction}

\section{Nutritional stress and pregnancy outcomes}

Suboptimal nutrition and maternal mental health during pregnancy have been linked to increased infant morbidity and mortality $[1,2]$. Although the placenta selectively supplies necessary nutrients throughout pregnancy, fetal growth is most vulnerable to maternal dietary deficiencies (e.g., protein and micronutrients) during placental development early in pregnancy [3]. Not only nutritional status, but also maternal mental health (stress, anxiety and depression) has been identified as a significant prenatal factor predicting postnatal development $[4,5]$. The time from conception to 2 years is when individuals are most sensitive to factors leading to chronic disease in adult life [6-8]. Where there is a lack of balanced nutrition, and maternal mental ill-health, it may severely affect fetal neuro-behavioural development [1]. 
One condition likely to cause nutritional deficiency is Hyperemesis gravidarum (HG). HG is defined by weight loss through excessive vomiting during pregnancy and plays a significant role in health outcomes [9, 10]. These effects are long term, in that in utero exposure to HG is significantly associated with poor postnatal mental health outcomes for the child [10]. Fejzo et al. [10] found a 3.6-fold increased risk of emotional and behavioural disorders of infants born to mothers who suffered from HG. Importantly, there was no effect on adult siblings who were not affected by HG compared with those exposed to $\mathrm{HG}$ prenatally. Given that only offspring from mothers who had a poor diet during the pregnancy caused by $\mathrm{HG}$ rather than their siblings brought up in the same environment showed negative emotional and behavioural effects, it seems likely that the negative effects are caused by HG and that HG has long-term consequences lasting into adulthood.

\section{Current study}

Although children of mothers with $\mathrm{HG}$ have been shown to be affected after birth [3, 6, 7, 11, 12], we know of no research on the health of the fetus. Given that $0.5-3 \%$ of pregnant women suffer from diagnosed HG [13, 14], the aim of this pilot study was to test whether $\mathrm{HG}$ is reflected in fetal health expressed in fetal movement profiles in later pregnancy, and whether the nutritional stress on the mother can be assessed using isotopic analysis of hair. There is a need for non-invasive measures to test effects of $\mathrm{HG}$ during pregnancy with the potential to establish not only growth parameters, but also cognitive and affective parameters $[5,15]$. With the advent of 4D ultrasound scanning, it is now possible to establish fetal movement profiles as a way of assessing their development. Such techniques have the potential for deriving diagnostic tools aimed at the early non-invasive detection of developmental dysfunction [15-19].

Although the effects of nutritional stress and mental stress have been reported in separate studies, their relative effects have not been tested. One way in which we can assess nutritional status is to use hair isotope analysis, yielding longitudinal information, from preconception through to the time of sampling at 36-weeks' gestation. Mental health measures, including stress, anxiety and depression can be assessed by standardized questionnaires. By combining both types of tests with the longitudinal data from 4D scans at 32- and 36-weeks' gestation, this study investigates for the first time both the effects of maternal nutritional status and maternal stress, anxiety and depression on the development of the fetus.

In this feasibility study, we examined fetal movement profiles of two groups of mothers, six mothers who had suffered HG and six healthy mothers, using 4D ultrasound scans recorded at 32- and 36-weeks' gestation. We tested for the presence of, and recovery from, under-nutrition catabolism in early pregnancy using maternal hair that grew from before conception to 36-weeks' gestation by comparing maternal carbon isotope ratios (expressed as $\delta^{13} \mathrm{C}$ ) and nitrogen isotope ratios $\left(\delta^{15} \mathrm{~N}\right)$ in their hair reflecting dietary inputs.

We hypothesized first that HG is significantly associated with catabolism as evidenced by hair samples and second that HG in the first trimester of pregnancy affects the fetal movement profile independent of maternal stress, depression and anxiety.

\section{Stable isotopes in hair}

The ratios of the stable isotopes of carbon $\left({ }^{13} \mathrm{C} /{ }^{12} \mathrm{C}\right)$ and nitrogen $\left({ }^{15} \mathrm{~N} /{ }^{14} \mathrm{~N}\right)$ vary in humans due to external variations in food sources and internal metabolic processes. By convention, the ratios are expressed as fractional deviations from the ratios of standard materials, with the notation $\delta^{13} \mathrm{C}$ and $\delta^{15} \mathrm{~N}$, and given as parts per thousand (per mille, \%o) [20]. The major sources of external variations for $\delta^{13} \mathrm{C}$ are differences in photosynthesis, with three classes of food relevant for humans. The majority of plants, and the animals that consume them, use the $\mathrm{C}_{3}$ photosynthetic process and produce foods with a mean $\delta^{13} \mathrm{C}$ of $-25 \%$. Some tropical grasses, including maize and sugarcane, use the $\mathrm{C}_{4}$ photosynthetic process and produce foods with a mean $\delta^{13} \mathrm{C}$ of $-13 \%$. In marine ecosystems, the dissolution of carbon dioxide into seawater decreases the $\delta^{13} \mathrm{C}$ to a mean of $-17 \%$. Variations in $\delta^{15} \mathrm{~N}$ are mainly driven by changes at each step in the food chain, with $\delta^{15} \mathrm{~N}$ values increasing by $3-5 \%$ o for each step in the food chain, starting with atmospheric nitrogen at $0 \%$, and increasing through plants, herbivores, omnivores, carnivores and super-carnivores. Food chains in marine and freshwater ecosystems tend to be longer than in terrestrial ecosystems so that fish can have higher $\delta^{15} \mathrm{~N}$ than terrestrial animals.

At homeostasis with a consistent diet, protein in a consumer body has $\delta^{15} \mathrm{~N}$ and $\delta^{13} \mathrm{C}$ higher than diet by 3-5\%o and $0-1 \%$, respectively, while body fat has a lower $\delta^{13} \mathrm{C}$ than body proteins by about 5\%o [21]. During growth, (including weight gain in pregnancy) positive nutrient balance is predicted to slightly lower both $\delta^{13} \mathrm{C}$ and $\delta^{15} \mathrm{~N}$ in newly formed tissues, as intake of foods with lower ratios than the body exceeds output of waste. During weight loss, catabolism of the body's own tissues occurs, drawing on protein for nitrogen and partly on fats for carbon. New tissues formed during weight loss are thus expected to have raised $\delta^{15} \mathrm{~N}$ and lowered $\delta^{13} \mathrm{C}$ compared to those formed under homeostasis. The changes in $\delta^{13} \mathrm{C}$ and $\delta^{15} \mathrm{~N}$ during catabolism and recovery, when plotted against time, typically show opposing covariance [22, 23], with diverging and then converging values, a feature informally termed 'bubble'. 
Table 1 Participant variables $(N$ indicates the number of participants for whom we could obtain this information)

\begin{tabular}{lll}
\hline & Healthy group & HG group \\
\hline Maternal age & $N(6)$ & $N(6)$ \\
& $M=24.17$ & $M=26.67$ \\
Level of education & SD-5.04 & SD $=4.18$ \\
& 3 to GCSE level & 1 to GCSE level \\
& 2 to College/A-level & 3 to College/A-level \\
Fetal head circumference at & 1 to Bachelor's degree level & 1 to Bachelor's degree level \\
20 weeks & $N(5)$ & 1 to Master's degree level \\
& $M=16.72$ & $N(6)$ \\
Birth weight & $\mathrm{SD}=9.58$ & $M=17.43$ \\
& $N(6)$ & $\mathrm{SD}=4.64$ \\
Apgar score 1 min & $M=3483$ & $N(6)$ \\
& $\mathrm{SD}=750.26$ & $M=3451.50$ \\
Apgar score 5 min & $N(5)$ & $\mathrm{SD}=282.03$ \\
& $M=8.80$ & $N(4)$ \\
Fetus sex & $\mathrm{SD}=.45$ & $M=8.25$ \\
& $(N=5)$ & $\mathrm{SD}=1.5$ \\
& $M=9$ & $N(4)$ \\
& $\mathrm{SD}=.0$ & $M=9$ \\
& $\mathrm{Two}$ boys, four girls & $\mathrm{SD}=.82$ \\
& & Three boys, three girls
\end{tabular}

These predictions have been borne out in small-scale studies of hair isotopes in humans. A study of ten pregnant women showed $\delta^{15} \mathrm{~N}$ correlated with weight gain but detected no change in $\delta^{13} \mathrm{C}[8]$. However, $\delta^{15} \mathrm{~N}$ was shown to increase during $\mathrm{HG}$ in a study of eight pregnant women [8], also with no detectable change in $\delta^{13} \mathrm{C}$ (their Table 2 shows mean change $+0.1 \pm 0.1 \% o$ ). Two studies of recovering anorexics, with six [22] and seven [23] participants, showed declining $\delta^{15} \mathrm{~N}$ and increasing $\delta^{13} \mathrm{C}$ as body mass increased. After the initial changes, some of these individuals showed other patterns due to significant changes in diet, such as vegetarian to omnivorous. Another study of 13 deceased individuals who suffered a period of severe starvation shortly prior to death showed decreasing $\delta^{13} \mathrm{C}$ and increasing $\delta^{15} \mathrm{~N}$ [24]. Assuming no isotopic change in the diet, the expected isotopic changes in hair during a pregnancy with normal weight gain are therefore declines in both $\delta^{15} \mathrm{~N}$ and $\delta^{13} \mathrm{C}$, but, in contrast, during $\mathrm{HG}$ hair is expected to show increasing $\delta^{15} \mathrm{~N}$ and declining $\delta^{13} \mathrm{C}$. With recovery from $\mathrm{HG}, \delta^{15} \mathrm{~N}$ should decrease and $\delta^{13} \mathrm{C}$ increase in hair, followed by both decreasing when the pregnancy returns to normal. The changes in $\delta^{13} \mathrm{C}$, however, may be

Table 2 Observed variables

\begin{tabular}{lllll}
\hline Variable & Healthy sample 32 weeks & Healthy sample 36 weeks & HG sample 32 weeks & HG sample 36 weeks \\
\hline HADS anxiety & $N=6$ & $N=6$ & $N=6$ & $N=6$ \\
& $M=3.67$ & $M=3$ & $M=11.17$ & $M=10.67$ \\
HADS depression & $\mathrm{SD}=1.86$ & $\mathrm{SD}=3.11$ & $\mathrm{SD}=5.74$ & $\mathrm{SD}=6.38$ \\
& $N=6$ & $N=6$ & $N=6$ & $N=6$ \\
& $M=2.67$ & $M=3.17$ & $M=11.17$ & $M=11.17$ \\
Total maternal attachment & $\mathrm{SD}=1.86$ & $\mathrm{SD}=2.64$ & $\mathrm{SD}=2.23$ & $\mathrm{SD}=3.49$ \\
& $N=6$ & $N=6$ & $N=6$ & $N=6$ \\
PUQE & $M=83.67$ & $M=85.83$ & $M=82.83$ & $M=83.00$ \\
& $\mathrm{SD}=8.55$ & $\mathrm{SD}=3.92$ & $\mathrm{SD}=6.37$ & $\mathrm{SD}=8.74$ \\
PSS & $N=6$ & $N=6$ & $N=6$ & $N=6$ \\
& $M=3.17$ & $M=3.67$ & $\mathrm{SD}=3.37$ & $M=6.83$ \\
& $\mathrm{SD}=.41$ & $\mathrm{SD}=1.21$ & $N=6$ & $\mathrm{SD}=4.31$ \\
Mean fetal exact age at scan & $N=6$ & $M=6$ & $M=23.00$ & $N=6$ \\
& $M=9.33$ & $\mathrm{SD}=5.13$ & $\mathrm{SD}=4.78$ & $M=20$ \\
& $\mathrm{SD}=5.61$ & $N=6$ & $N=6$ & $\mathrm{SD}=7.87$ \\
& $M=227$ (days) & $M=255$ (days) & $M=228$ (days) & $M=6$ \\
\end{tabular}


small or close to zero, and may also be hard to detect in the presence of other factors such as measurement uncertainty and variations in diet.

\section{Methods}

\section{Participants}

Twelve women, whose fetuses were determined healthy at their 20-week anomaly scan, and who had a healthy pre-pregnancy BMI, were recruited through opportunity sampling. Two groups of pregnant women were recruited: six women having a healthy pregnancy with normal weight gain and six women who were diagnosed with HG by a medical practitioner and had lost at least $10 \%$ of their body weight in the first trimester of pregnancy (see Tables 1 and 2 ). However, because the recruitment protocol required a healthy 20 -week anomaly scan, we did not record weight loss data in the first trimester. All participants were white British from the North East of England. All women underwent 4D ultrasound scanning at the Windows to the Womb Clinic, Gateshead, UK by experienced sonographers, lasting around 15-20 min at 32- and 36-weeks' gestation. Fetal fine-grained mouth movements were coded frameby-frame using the Fetal Observable Movement System (FOMS) $[15,16]$. The scanning took place with mothers lying in a darkened room on their back or on their side, depending on the position of the fetus in the womb and on the mother's comfort. The fetal face and upper torso were visualized by means of 4D full frontal and facial profile ultrasound recordings, as well as traditional 2D images. The scans were recorded for off-line analysis with a GE Voluson E8 or E10 Expert Ultrasound System using a RAB6-RS transducer. Mothers received a DVD copy of their scans. Mothers completed the Perceived Stress Scale (PSS) questionnaire, assessing stress levels at each scan $[25,26]$. The PSS is a widely used valid and reliable tenitem five-point Likert-based scale measuring the degree to which mothers perceive their life as stressful (ranging from $0=$ 'no stress' experienced during the last month to $4=$ 'very often' stressed). Additionally, mothers completed the Hospital Anxiety and Depression Scale (HADS; with two sub-scales HADS-A refers to anxiety and HADS-D refers to depression) [27]. The scale has 14 items [seven items for anxiety and seven for depression and ranges from 0 (minimum) to 21 (maximum)]. Mothers also completed the Pregnancy-Unique Quantification of Emesis/Nausea (PUQE) index resulting in mild (score $\leq 6)$, moderate (score $=7-12$ ), and severe (score $\geq 13$ ) [28] and an attachment scale [29].

\section{Hair sample analysis}

Nutritional status was tested with hair, at least $10 \mathrm{~cm}$ in length collected at 36-weeks' gestation in both the HG and heathy groups of women. Human hair grows at an average of $1 \mathrm{~cm}$ per month [30,31] therefore collection of $10 \mathrm{~cm}$ long hair at 36 weeks allows month by month analysis of carbon and nitrogen isotopes from preconception through to 36-weeks' gestation. A group of ten or more hairs were bound with adhesive tape to keep them aligned before cutting as close to the scalp as possible. Hair samples were coded with anonymous codes. Each bundle of hair was cut into $1 \mathrm{~cm}$ segments, corresponding approximately to growth from 2 months prior to conception up to 8 months of gestation. The $1 \mathrm{~cm}$ segments were placed in individual micro tubes, and prepared using a modification of previous protocols [22]. To remove fats from the hair, samples were washed three times by ultrasonication in $1 \mathrm{ml}$ of $2: 1$ methanol: chloroform. The solvent was removed by ultrasonication in $1 \mathrm{ml}$ of deionised water and the samples dried at $55^{\circ} \mathrm{C}$.

Samples were analysed by IsoAnalytical Ltd (Crewe, Cheshire). After weighing into tin capsules, samples were combusted on a Europa Scientific elemental analyser, and isotope ratios measured on a Europa Scientific 20-20 Isotope Ratio Mass Spectrometer. The reference material was IA-R068 (soy protein, $\delta^{13} \mathrm{C}_{\mathrm{VPDB}}=-25.22$ $\%$ o, $\delta^{15} \mathrm{~N}_{\mathrm{AIR}}=0.99 \%$ ), and quality control check samples were IA-R068, IA-R038 (L-alanine, $\delta^{13} \mathrm{C}_{\mathrm{VPDB}}=-24.99$ $\% o, \quad \delta^{15} \mathrm{~N}_{\mathrm{AIR}}=-0.65 \%$ ), IA-R069 (tuna protein, $\delta^{13} \mathrm{C}_{\mathrm{VPDB}}=-18.88 \% o, \delta^{15} \mathrm{~N}_{\mathrm{AIR}}=11.60 \%$ o $)$ and a mixture of IAEA-C7 (oxalic acid, $\delta^{13} \mathrm{C}_{\mathrm{VPDB}}=-14.48 \%$ ) and IA-R046 (ammonium sulfate, $\delta^{15} \mathrm{~N}_{\mathrm{AIR}}=22.04 \%$ ), which are calibrated against the international standards IAEA-CH-6 and IAEA-N-1. Every fifth sample was measured in duplicate. The technical error of measurement from the duplicate samples was $0.06 \%$ for $\delta^{13} \mathrm{C}$ and $0.05 \%$ for $\delta^{15} \mathrm{~N}$.

Using conventional methods, catabolic and non-catabolic isotope profiles were classified by eye. The anonymized data series for each woman was classified by one of the authors (Millard), without knowledge of their HG status, as showing evidence for catabolism, where there was a clear opposing covariance in the data, possible evidence for catabolism, where there was less clear evidence for opposing covariance, or no evidence for catabolism, where there was no opposing covariance.

\section{Statistical analysis}

The data consist of repeated measures, with each fetus scanned for 15-20 min at 32- and 36-weeks' gestation and fetal fine-grained movements coded frame by frame. The total number of movements serves as dependent variable, which is related through a log-linear model to explanatory 
variables including stress, depression and anxiety scores as well as effects of gestational week and HG. Further predictors including maternal age, fetal age and education level were initially considered (see supplementary information S3) but removed from the analyses, which follow, as they did not carry significant effects. Since the observed codable scan length varied between fetuses, the model further includes an offset of log codable scan length, thus, effectively modelling total movement rate. Finally, there is a random effect term assessing between fetus variability. The model can hence be formulated as $(H)$ :

$$
\begin{aligned}
\log \lambda_{\mathrm{it}}= & \log \left(\text { scan length }_{\mathrm{it}}\right)+\beta_{0} \beta_{1} \text { gestational age }_{\mathrm{it}} \\
& +\beta_{2} \mathrm{HG}_{i}+\beta_{3} \times \mathrm{HG}_{i} \text { gestational age }_{\mathrm{it}} \\
& +\beta_{4} \mathrm{PSS}_{\mathrm{it}}+\beta_{5} \mathrm{HADS}_{-} A_{\mathrm{it}}+\beta_{6} \mathrm{HADS}_{-} D_{\mathrm{it}}+u_{i}
\end{aligned}
$$

where the total number of movements $C_{i t}$ of fetus $i=1, \ldots, 12$ at time $t=32,36$ follows a negative Binomial distribution

$C_{i t} \sim \mathrm{NB}\left(\lambda_{i t}, \theta\right)$

and the random effect a Gaussian distribution

$u_{i} \sim \operatorname{Normal}\left(0, \sigma_{u}^{2}\right)$

The three predictor variables in the final row of $(H)$ are continuous variables, while HG is a two-level-factor variable indicating the health status, that is the absence $(=0)$ or presence $(=1)$ of HG. The variable GestationalAge could be described by either a continuous variable or a two-level factor for weeks 32 or 36 . For ease of interpretation, we work with the latter in this paper, with week 32 as reference category. The conclusions do not change if the continuous version is used. We will also, later, consider a similar model $(C)$ in which HG is replaced by the two-level factor catabolism (with one level for "yes and possible" and one level for "no catabolism").

We have opted here for a Negative Binomial response model rather than Poisson as in comparable studies [16, $32,33]$, since over dispersion for these data was relatively strong. The $\theta$ parameter takes values of 4.25 and 3.44 in models $(H)$ and $(C)$, respectively, where $\theta \rightarrow \infty$ would correspond to "no over dispersion". The corresponding likelihood ratio (LR) tests favour the Negative Binomial model with $p$ values $<0.0001$ in each case. Hence, using the Poisson response for these data would lead to incorrect standard errors with overstated significances. We fitted the model using the glmmTMB function in the glmmTMB library in R [34]. The significance of model parameters was assessed by Wald test $p$ values on the parameter coefficients, with $p$ values less than 0.05 considered significant.

\section{Results}

All 24 movement scans were successful typically yielding 8-12 min of codable scan allowing rates to be determined. Variations in length of coding were statistically controlled. Reliability testing was conducted on $16 \%$ of the sample for which the mean Cohen's Kappa was 0.93 with a range of $0.83-0.98$.

Isotope profiles were obtained for all women (supplementary information S2). One subsample (3935-07) was rejected as showing a $C: N$ ratio too high for hair. Figure 1 shows examples of catabolic and non-catabolic profiles, and a full set of profiles is in supplementary information S1, together with their HG status and catabolism classification.

\section{Independent variable analysis}

Before modelling the fetal movement profiles, we conducted an explanatory analysis on the maternal factors: HG status, presence of catabolism, stress, depression and anxiety. There were strong interactions between these independent variables which were taken account of in the analysis. Despite these correlations, the variables may still have differential effects on the total number of movements.

\section{HG and catabolism}

The contingency table of catabolism and health status (Table 3) indicates an association between HG status and the presence of catabolism, which is confirmed by an unconditional exact test for $2 \times 2$ contingency tables, with $p=0.020$ [35]. There was an entire absence of catabolism for healthy mothers, which further strengthens the link between HG and catabolism.

\section{Nutritional stress and mental health}

Scores for stress, anxiety, and depression, are significantly related to health status (t-tests, each $p<0.001)$ and two scores to catabolism (PSS: $p=0.032$, HADS_D: $p=0.0015$ ), but not to gestational week (each $p>0.7$ ). The three scores are highly correlated with each other, with all correlation coefficients between 0.82 and 0.90 .

\section{Fetal movement analysis}

Table 4 shows estimates (non-italic rows), standard errors and $\mathrm{p}$ values of the fitted model $(H)$. All included parameters except PSS (stress) and HADS_A (anxiety) are significant at the $5 \%$ level. From the formulation of the model, it follows 
Fig. 1 Examples of isotope profiles from hair. a Profile indicating catabolism with rising and falling $\delta^{15} \mathrm{~N}$ without parallel changes in $\delta^{13} \mathrm{C}$. b Profile with no evidence for catabolism with broadly uniform or falling $\delta^{15} \mathrm{~N}$ and $\delta^{13} \mathrm{C}$

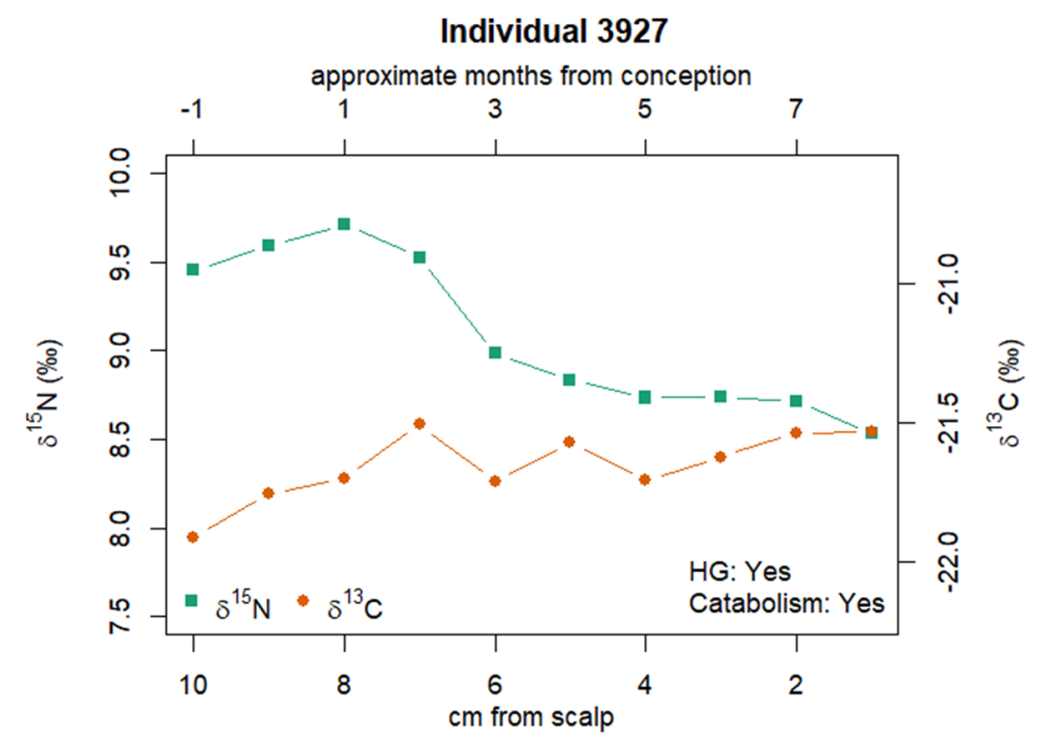

(a) Profile indicating catabolism with rising and falling $\delta^{15} \mathrm{~N}$ without parallel changes in $\delta^{13} \mathrm{C}$.

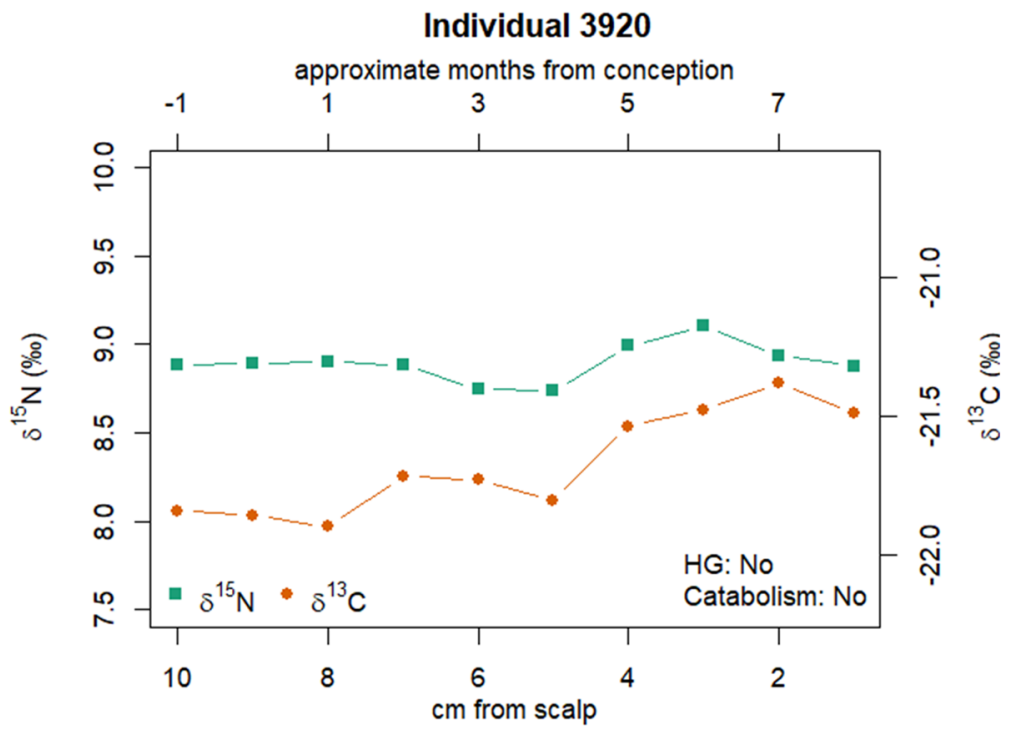

(b) Profile with no evidence for catabolism with broadly uniform or falling $\delta^{15} \mathrm{~N}$ and $\delta^{13} \mathrm{C}$.
Table 3 Contingency table of health status and catabolism frequencies

\begin{tabular}{lll}
\hline Catabolism & Health status \\
\cline { 2 - 3 } & Healthy & Hyperemesis \\
\hline No & 6 & 2 \\
Yes/possible & 0 & 4 \\
\hline
\end{tabular}

Reported frequencies refer to individual fetuses; there are hence $2 \times 12=24$ total scans coded frame by frame and measurements reported that the coefficients have an exponential and multiplicative impact on the movement per scan length rate. Several aspects of the model deserve further discussion.

\section{HG effects}

We see that at week 32, after adjusting for stress, anxiety, and depression, the movement rate is larger by a factor of $\exp (1.4507)=4.27$ for the $\mathrm{HG}$ group compared with the healthy group. From Table 4, we know that this a significant effect ( $p=0.008$, Cohen's $d=1.34$ ), which is also clearly visible by the large gap in the left-hand side in Fig. 2a. In contrast, at week 36, the difference between 
Table 4 Coefficient estimates of model $(H)$

\begin{tabular}{lllll}
\hline Coefficient & Estimate & SE & $z$ value & $p$ value \\
\hline Intercept & -3.8996 & 0.3160 & -12.340 & $<2 \mathrm{e}-16$ \\
& -3.7136 & 0.2840 & -13.077 & $<2 e-16$ \\
HG & 1.4507 & 0.5470 & 2.652 & 0.0080 \\
& 1.0626 & 0.3896 & 2.727 & 0.0064 \\
GestationalAge & 0.7174 & 0.3413 & 2.102 & 0.036 \\
& 0.6364 & 0.3214 & 1.980 & 0.047 \\
HG:GestationalAge & -1.1946 & 0.4807 & -2.485 & 0.013 \\
& -1.3910 & 0.4440 & -3.133 & 0.0017 \\
PSS & 0.0645 & 0.0384 & 1.679 & 0.093 \\
HADS_A & 0.0463 & 0.0432 & 1.072 & 0.284 \\
HADS_D & -0.1999 & 0.0760 & -2.631 & 0.0085 \\
\hline
\end{tabular}

Italic rows refer to the model fitted without stress, depression and anxiety

healthy and HG groups is not significant ( $p=0.577$, Cohen's $d=-0.14)$. Also, for fetuses from the healthy group, from $\exp (0.717)=2.05$ one infers a two-fold increase in movement rate from week 32 to week 36 (this is the dashed line in Fig. 2a). However, if fetuses are from the HG group, the change from week 32 to week 36 is of magnitude $\exp (0.717$ )$\times \exp (-1.195)=0.620$, that is a reduction by $38 \%$, and this difference (between two-fold increase and a 38\% decrease) in the effect of gestational week for the two groups corresponds to the significant $(p=0.013)$ interaction term in Table 4.

\section{Catabolism effects}

If we replace the factor for $\mathrm{HG}$ in the analysis by catabolism, we would expect from previous considerations a similar picture. However, given the small sample size, the catabolism effect seems less pronounced than the reported HG diagnosis with the loss of $10 \%$ of body weight in the first trimester (see Fig. $2 \mathrm{~b}$ and supplementary information S4). The variables concerning catabolism and gestational group do not achieve significance either individually or jointly ( $p$ value of LR test 0.426 at 3 degrees of freedom $[d f]$ ). This changes only to a limited extent when not including the score variables (the $p$ value of a LR test for joint inclusion of catabolism, gestational age, and their interaction, is now at 0.185 at $3 d f$; with the $p$ value for catabolism by itself at $p=0.025$ ). We conclude that, albeit strongly associated with HG, catabolism as identified by hair samples seems to be underpowered
Fig. 2 Interaction plots. a Interaction plot for health status and gestational age; $\mathbf{b}$ interaction plot for catabolism and gestational age. Line endpoints correspond to means of the 'total number of movements divided by scan length', for the respective factor level combinations. The scan length is measured in seconds [s], so the vertical axis has the unit $1 / \mathrm{s}$ (a)

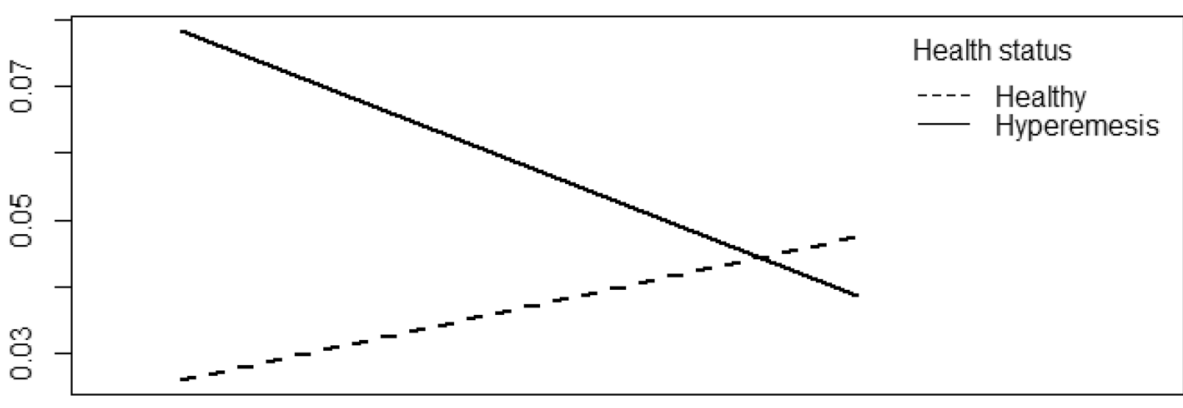

32 36

gestational age [weeks]

(b)

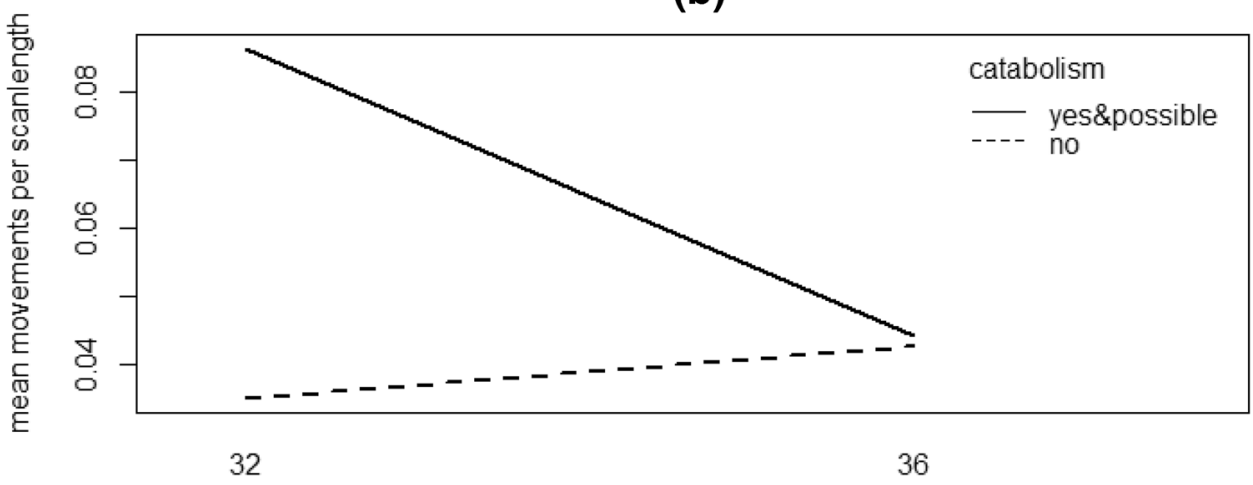

gestational age [weeks] 
and in this pilot study is overall a less useful predictor for fetal movements.

\section{Maternal mental health}

From the model output and the previously described collinearity of the score variables (HADS_A for anxiety, HADS_D for depression, and PSS for stress), we see that, not all these variables appear relevant to the model. Removing PSS and HADS_A from the model renders however HADS_D nonsignificant (not shown), and a likelihood ratio test for the joint inclusion of the three score variables against their exclusion gives moderate evidence towards their inclusion $(p=0.098$ at $3 d f)$. However, importantly, the main reason for inclusion of the score variables is to evaluate nutritional impact on fetal movements over and above the effects of anxiety, depression and stress. The effect size of the score variables for the model fitted in Table 4 is $\eta^{2}=0.472$.

\section{Distinct movement analysis}

So far, we have discussed the total number of movements as the only response variable. However, we have specific measurements available on ten distinct movements, which are lips part, lip pucker, lip pull, upper lip raiser, lower lip depressor, lip corner depressor, lip pressor, mouth stretch, lip stretch, and lip suck. Based on the current small sample sizes, we would consider it as unscientific to report specific $p$ values for each these (with multiple testings' problems unavoidably arising), therefore we just present the results of this analysis in graphical form (Fig. 3) through a heatmap and dendrograms. Each row of the rows in the heatmap corresponds to a fitted model, with the respective movement type as response, and the variables PSS, HADS-A, HADS-D, HG, gestational age, as well the interaction term between the latter, as predictor terms. The colour in the heatmap represents the absolute $z$ value of the estimated coefficients, where red means more evidence of relevance of each predictor variable for the respective movement type, and white less evidence. The graph also includes, in the first row of the heatmap, the total number of movements for reference.

Notably, the strongest HG effects are evident with the total number of movements, lip parting and lip puckering. Further promising directions for future research emerge from the graph; for instance, fetal lip pulling appears to be strongly related to maternal stress, anxiety, and depression. Lip pressing appears unexplained by any variable. The frequency of lip stretch, and lip suck is most strongly affected by the gestational week. It is useful to consider these results in conjunction with the movement-specific interaction plots

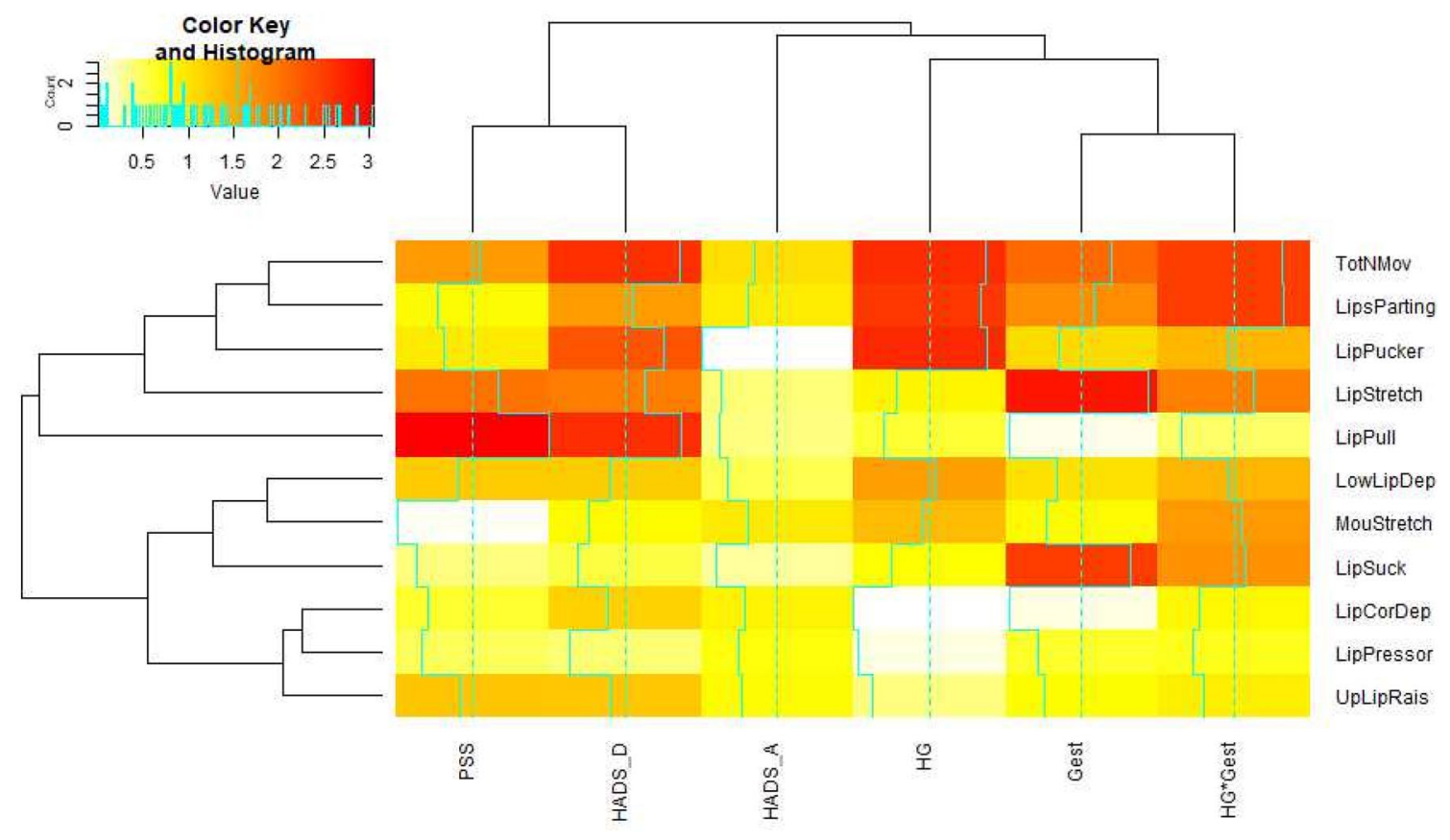

Fig. 3 Heatmap with dendrogram for individual movements. The colour strength from red to white corresponds to the strength of effects with deep red the strongest effect, and white smallest effect. The strength of the effect can also be assessed through the light-blue stepshaped curves, with the vertical dashed lines indicating a $z$ value of 2 on a standardized Gaussian scale. On the horizontal axis, Gest cor- responds to gestational age, and $\mathrm{HG} \times \mathrm{Gest}$ to the interaction effect of gestational age and health status. On the vertical axis, the following abbreviations are used: LowLipDep lower lip depressor, MouStretch mouth stretch, LipCorDep lip corner depressor, UpLipRais upper lip raiser, TotNMov total number of movements 
for the effects of HG and gestational week (supplementary information S5). These plots show that, throughout all movement types, the most dominant feature is again the decrease in the number of movements from week 32 to week 36 for the HG group, corresponding to the generally darker shades in the last column of Fig. 3. This decrease is visible in S5 for seven out of ten movements in absolute terms, and for all ten movements relative to the healthy group.

\section{Discussion and conclusion}

\section{HG affects fetal movement}

In this study, we examined the effects of HG on the nutritional profile of the mother by testing maternal hair for carbon isotope ratios $\left(\delta^{13} \mathrm{C}\right)$ and nitrogen isotope ratios $\left(\delta^{15} \mathrm{~N}\right)$ in hair reflecting maternal diet. At 32-weeks' gestation, fetuses with mothers having HG showed significantly higher rate of fetal movements compared with fetuses of healthy mothers. On the one hand, significantly decreased fetal movements signpost an increased risk of fetal growth restriction, stillbirth, preterm birth and the need of emergency caesarean section [36]. Donker et al. [37] suggested that a significant reduction in the range of different types of specific movement patterns, such as frequency of jaw opening or neck turn, are an indication that fine-grained movement profiles might be helpful in identifying distress. On the other hand, recent research by Heazell et al. [38] argues that maternal heightened anxiety, fetal seizures or unexplained fetal insult might lead to abnormally increased fetal movement profiles and stillbirth occurring after 37-weeks' gestation. In the current study, movement profiles of fetuses of mothers with HG showed an increased movement profile at 32-weeks' gestation and this was followed by the rapid decline of fine-grained mouth movements over the next 4 weeks to slightly (albeit nonsignificantly) below that of healthy fetuses. This movement profile at 36 weeks can be interpreted as either leading to normalized movement profiles or potentially to abnormally low frequencies of movements, which are themselves an indicator of an unhealthy development [36]. These interpretations might be distinguished using a scan later in pregnancy.

In sum, fine-grained movement analysis might be a helpful tool in identifying distress as well as normal development. Reissland et al. [15] and Castiello and Parma [39] argue that fetal movement profiles are potential tools to investigate prenatal cognition and the pre- to post-natal continuity of cognitive development including "anticipation" in the fetus. Hence, not only gross body movements, but also more fine-grained movement analyses might be usefully employed to assess healthy fetal development. In previous research, fetal movements tested included general body movements, startle and twitch movements, isolated limb movements, breathing movements, hiccups, isolated head and neck movements, sucking and swallowing, jaw movements (including yawning), hand-face contact, stretch and rotation [40, 41], as well as fine-grained fetal facial movements [42]. The analysis of gross body movements is a less conclusive measure of fetal distress compared to the finegrained analysis utilised in this study. The heatmap (Fig. 3) indicates that there might be potential to use fine-grained movement analysis to highlight specific movements, such as lip parting and lip puckering, which may be predictive of fetal state. Furthermore, other specific movements (e.g., lip pull) might be influenced by maternal mental health which has previously been shown indicative of neurobehavioral development, such as increased eye-blink rate in fetuses of anxious mothers [5]. These specific movements could potentially be used as prenatal markers for adverse neurodevelopment.

\section{Hyperemesis is detectable in hair isotopes}

Results indicated that the majority of mothers suffering from hyperemesis had either suspected or confirmed catabolic profiles in their first trimester hair samples. Our results confirm one other study showing that HG is evidenced in the stable isotopes of hair [8] by an increase in $\delta^{15} \mathrm{~N}$. HG mothers in the current study had experienced significant weight loss, which added to the power of our study, compared to the 'restricted weight gain and/or weight loss' previously studied [8], but our study lacks information from monitoring of diet and weight throughout pregnancy which could improve our understanding of isotopic changes. The current results suggest that with more extreme weight loss decreases in $\delta^{13} \mathrm{C}$ are also detectable. We also made no allowance for the fact that only $85-90 \%$ of scalp hairs are actively growing at any time and there is therefore smoothing of the isotopic signal when multiple hairs are sampled [43], but this could only be assessed using plucked rather than cut hairs. Catabolic and non-catabolic isotope profiles are currently identified by eye and classified in a binary fashion. However, the measurements are continuous data and, in the future, it would be useful to develop a quantified scale computed from the data to express graduations in dietary stress that mothers have experienced. This would allow more subtle effects on fetal development to be investigated, and the retrospective nature of their identification would reduce the confounding effect of unrecorded HG or morning sickness in studies of fetal development.

\section{HG and postnatal development}

This interpretation of our prenatal data is strengthened by work on long-term effects of maternal HG on their offspring. 
Parker et al. [44] report long-term effects of children from 5-12 years in terms of poorer scores on several neurodevelopmental measures as well as psychosocial problems including internalizing and externalizing behaviours of children exposed to HG prenatally. The effects of HG seem to be long term beyond childhood. Mullin et al. [12] found when comparing emotional and behavioural outcomes of adults who had been exposed to HG during their fetal life compared with healthy pregnancies, those who were exposed to HG prenatally were significantly more likely to be diagnosed with depression, and anxiety as well as bipolar disorders in adulthood. Additionally, HG seemed to be a significant factor indicating that this condition needs to be treated not only prenatally, but that follow-up of the children might be called for. In sum, although the effects of HG are not apparent in terms of birthweight, Apgar scores or gestational age at delivery [45] there is evidence of postnatal effects, which warrant more investigation.

The current pilot study indicates that HG affects the normal development of fetuses, and that this is evident from as early as 32-weeks' gestation. More research is needed on the prevailing effects of this altered developmental trajectory. Additionally, a larger fetal study with a postnatal follow-up is needed to use these differentiated movement profiles in HG fetuses as potential markers for later neurodevelopmental outcomes.

Author contributions All authors have participated this paper with the following input to it: NR: protocol development, data management, data analysis, manuscript writing and editing. ARM: protocol development, hair sample analysis, manuscript writing and editing. RW: data coding, creating SPSS data file, manuscript editing. BU: data coding, creating SPSS data file. CM: recruitment, data collection. SF: data collection and coding. JE: statistical analyses, manuscript writing and editing. All authors have approved the final version of the ms.

Funding The project received Seedcorn funding from Durham University 16_05-18.

\section{Compliance with ethical standards}

Conflict of interest I certify that no actual or potential conflict of interest in relation to this article exists.

Ethical approval This study was conducted in accordance with the Declaration of Helsinki. Ethical permission for this study was obtained from Durham University (PSYCH-2018-04-03T12:06:04-dps0nr). All mothers gave informed written consent.

Informed consent I certify that all mothers gave informed written consent for the study.

Open Access This article is licensed under a Creative Commons Attribution 4.0 International License, which permits use, sharing, adaptation, distribution and reproduction in any medium or format, as long as you give appropriate credit to the original author(s) and the source, provide a link to the Creative Commons licence, and indicate if changes were made. The images or other third party material in this article are included in the article's Creative Commons licence, unless indicated otherwise in a credit line to the material. If material is not included in the article's Creative Commons licence and your intended use is not permitted by statutory regulation or exceeds the permitted use, you will need to obtain permission directly from the copyright holder. To view a copy of this licence, visit http://creativecommons.org/licenses/by/4.0/.

\section{References}

1. Vohr BR, Poggi Davis E, Wanke CA, Krebs NF (2017) Neurodevelopment: the impact of nutrition and inflammation during preconception and pregnancy in low-resource settings. Pediatrics 139:38-49

2. Glover V, O’Donnell KJ, O’Connor TG, Fisher J (2018) Prenatal maternal stress, fetal programming, and mechanisms underlying later psychopathology- A global perspective. Dev Psychopathol 30:843-854

3. Barker D, Clark P (1997) Fetal undernutrition and disease in later life. Rev Reprod 2:105-112

4. Glover V (2015) Prenatal stress and its effects on the fetus and the child: possible underlying biological mechanisms. Adv Neurobiol 10:269-283

5. Reissland N, Froggatt S, Reames E, Girkin J (2018) Effects of maternal anxiety and depression on fetal neuro-development. J Affect Disord 241:469-474

6. Adair LS (2014) Longterm consequences of nutrition and growth in early childhood and possible preventive interventions. Nestle Nutr Inst Workshops Ser 78:111-120

7. Fall C, Yajnik CS, Rao S, Davies AA, Brown N, Farrant HJ (2003) Micronutrients and fetal growth. J Nutr 13:1747-1756

8. Fuller BT, Fuller JL, Sage NE et al (2005) Nitrogen balance and $\delta^{15} \mathrm{~N}$ : why you're not what you eat during nutritional stress. Rapid Commun Mass Spectrom 19(18):2497-2506

9. Fejzo MS, Schoenberg FP, Macgibbon K et al (2016) Longterm health effects in children exposed in utero to hyperemesis gravidarum. Clin Obstet Gynecol Reprod Med 2(2):150-154

10. Fejzo M, Kam A, Laguna A, MacGibbon K, Mullin P (2019) Analysis of neurodevelopmental delay in children exposed in utero to hyperemesis gravidarum reveals increased reporting of autism spectrum disorder. Reprod Toxicol 84:59-64

11. Fejzo MS, Magtira A, Schoenberg FP et al (2015) Neurodevelopmental delay in children exposed in utero to hyperemesis gravidarum. Eur J Obstet Gynecol Reprod Biol 189:7984

12. Mullin PM, Bray A, Schoenberg F, MacGibbon KW et al (2011) Prenatal exposure to hyperemesis gravidarum linked to increased risk of psychological and behavioral disorders in adulthood. J Dev Orig Health Dis 2(4):200-204

13. Lacasse A, Rey E, Ferreira E et al (2009) Epidemiology of nausea and vomiting of pregnancy: prevalence, severity, determinants, and the importance of race/ethnicity. BMC Pregnancy Childbirth 9:26-35

14. Zhang J, Cai WW (1991) Severe vomiting during pregnancy: antenatal correlates and fetal outcomes. Epidemiology 2:454-457

15. Reissland N (2014) What the fetal face can tell us a discussion of the evidence, implications and potential for further research. Donald School J Ultrasound Obstet Gynecol 8:336-343

16. Reissland N, Francis B, Buttanshaw L (2016) The fetal observable movement system (FOMS). In: Reissland N, Kisilevsky B (eds) Fetal development research on brain and behavior, environmental influences, and emerging technologies. Springer, Berlin, pp 153-176 
17. Kurjak A, Carrera JM, Stanojevic M et al (2004) The role of 4D ultrasound in the neurological assessment of early human development. Ultrasound Rev Obstet Gynecol 4:148-159

18. AboEllail MAM, Hata T (2017) Fetal face as important indicator of fetal brain function. J Perinat Med 45(6):729-736

19. Reissland N, Francis B, Buttanshaw L et al (2016) Do fetuses move their lips to the sound that they hear? Pilot Feasibility Stud 2:14

20. Coplen TB (2011) Guidelines and recommended terms for expression of stable-isotope-ratio and gas-ratio measurement results. Rapid Commun Mass Spectrom 25:2538-2560. https://doi. org/10.1002/rcm.5129

21. Lee-Thorp JA, Sealy JC, van der Merwe NJ (1989) Stable carbon isotope ratio differences between bone collagen and bone apatite and their relationship to diet. J Archaeol Sci 16:585-599

22. Mekota A-M, Grupe G, Ufer S, Cuntz U (2006) Serial analysis of stable nitrogen and carbon isotopes in hair: monitoring starvation and recovery phases of patients suffering from anorexia nervosa. Rapid Commun Mass Spectrom 20:1604-1610. https:// doi.org/10.1002/rcm.2477

23. Mekota AM, Grupe G, Ufer S, Cuntz U (2009) Identifying starvation episodes using stable isotopes in hair. Rechtsmedizin 19:431. https://doi.org/10.1007/s00194-009-0630-3

24. Neuberger FM, Jopp E, Graw M, Püschel K, Grupe G (2013) Signs of malnutrition and starvation-reconstruction of nutritional life histories by serial isotopic analyses of hair. Forensic Sci Int 226:22-32. https://doi.org/10.1016/j.forsciint.2012.10.037

25. Cohen S, Kamarck T, Mermelstein R (1983) A global measure of perceived stress. J Health Soc Behav 24:386-396

26. Cohen S, Williamson G (1988) Perceived Stress in a Probability Sample of the United States. In: Spacapan S, Oskamp S (eds) The Social Psychology of Health. Sage Publications, Newbury Park, pp 31-67

27. Zigmond AS, Snaith RP (1983) The hospital anxiety and depression scale. Acta Psychiatrica Scandinavia 67(6):361-370

28. Koren G, Boskovic R, Hard M et al (2002) (PUQE) (pregnancyunique quantification of emesis and nausea) scoring system for nausea and vomiting of pregnancy. Am J Obstet Gynecol 186(2):228-231

29. Condon JT, Cokindale CJ (1998) The assessment of parent-toinfant attachment: development of a self-report questionnaire instrument. J Reprod Infant Psychol 16:57-76

30. O'Connell TC, Hedges REM (1999) Investigations into the effect of diet on modern human hair isotopic values. Am J Phys Anthropol 108:409-425

31. O'Connell TC, Hedges REM, Healey MA, Simpson AHRW (2001) Isotopic comparison of hair, nail and bone: modern analyses. J Archaeol Sci 28:1247-1255

32. Reissland N, Francis BJ, Mason J (2012) Development of fetal yawn compared with non-yawn mouth openings from
24-36 weeks gestation. PLoS ONE 7(11):e50569. https://doi. org/10.1371/journal.pone.0050569

33. Reissland N, Francis B, Aydin E et al (2013) The development of anticipation in the fetus: a longitudinal account of human fetal mouth movements in reaction to and anticipation of touch. Dev Psychobiol 56:955-963

34. Brooks ME, Kristensen K, van Benthem KJ, et al (2017) Modelling zero-inflated count data with glmmTMB. bioRxiv, https:// www.biorxiv.org/content/10.1101/132753v1. Accessed $26 \mathrm{Feb}$ 2020

35. Calhoun P (2016) Exact: unconditional exact test. R package version 1.7. Cran.R. https://CRAN.R-project.org/package=Exact29. Accessed June 2019

36. Olesen AG, Svare JA (2007) Decreased fetal movements: background, assessment, and clinical management. Acta Obstetrica Gynecologica Scandinavia 83:818-826

37. Donker ME, Eijckelhof BH, Tan G, de Vries JI (2009) Serial postural and motor assessment of fetal akinesia deformation sequence (FADS). Early Human Dev 85:785-790

38. Heazell AEP, Stacey T, O'Brien LM, Mitchell EA, Warland J (2018) Excessive fetal movements are a sign of fetal compromise which merits further examination. Med Hypotheses 111:19-23

39. Castiello U, Parma V (2018) Fetal kinematics: basic outcomes and translational outlook. ACS Chem Neurosci 9:165-166

40. Kurjak A, Stanojevic M, Andonotopo W et al (2005) Fetal behavior assessed in all three trimesters of normal pregnancy by fourdimensional ultrasonography. Croat Med J 46:772-780

41. De Vries JIP, Visser GHA, Prechtl HFR (1982) The emergence of fetal behaviour. I Qualitative aspects Early Human Development 7:301-322

42. Reissland N, Francis B, Mason J, Lincoln K (2011) Do facial expressions develop before birth? PLoS ONE 6(8):e24081. https ://doi.org/10.1371/journal.pone.0024081

43. Williams LJ, White CD, Longstaffe FJ (2011) Improving stable isotopic interpretations made from human hair through reduction of growth cycle error. Am J Phys Anthropol 145:125-136. https ://doi.org/10.1002/ajpa.21479

44. Parker SE, Starr JR, Collett BR (2014) Nausea and vomiting during pregnancy and neurodevelopmental outcomes in offspring. Paediatr Perinat Epidemiol 28(6):527-535

45. Agmon N, Sade S, Periente G et al (2019) Hyperemesis gravidarum and adverse pregnancy outcomes. Arch Gynecol Obstet 300:347-353

Publisher's Note Springer Nature remains neutral with regard to jurisdictional claims in published maps and institutional affiliations. 